\title{
Rotary Wheel Atomizer Study Using Computational Fluid Dynamics and Full-Scale Testing
}

\author{
Joensen, T..$^{\text {; }}$ Kuhnhenn, M. ${ }^{\mathbf{b}}$; Vinther, F..$^{\mathbf{a}^{*}}$; Reck, M. ${ }^{\mathrm{a}}$; Tropea, C.. \\ ${ }^{a}$ GEA Process Engineering A/S. Soeborg, Denmark \\ b Institute of Fluid Mechanics and Aerodynamics. Technische Universität Darmstadt, Darmstadt, \\ Germany.
}

*E-mail of the corresponding author: frank.vinther@gea.com

\begin{abstract}
This study models the internal fluid flow from the center to the edge of a rotary atomizer wheel, the flow out of the atomizer, including the film, rivulet and ligament formation, as well as the subsequent atomization process associated with the atomizer outflow using computational fluid dynamics with a volume of fluid approach.

The model shows how fluid exits through the overflow and not through the bushing at high inlet fluxes and can reproduce experimental results of power consumption. Furthermore, the drop-size distribution at a given distance from the bushing exit is in good agreement with experimental results.
\end{abstract}

Keywords: Spray drying; Rotary Atomizer; CFD; Droplet size; Volume of Fluid. 


\section{Introduction}

Feed atomization is one of the most important process steps in spray drying. In the chemical industry, rotary atomizers are often used because they are flexible, can handle abrasive materials and operate at very large capacities. Although development of atomizer wheels is usually based on a trial and error approach current designs are very advanced. New tools, like tailored computer simulations, are therefore needed to design a new and significantly improved generation of atomization wheels. These wheels will reduce atomizer power consumption, suffer less wear and can operate at higher capacity while avoiding overflow as described below. All this while improving the droplet size distribution which is the most important output from the atomizer. The size distribution influences every downstream aspect of the powder production process, ranging from drying chamber depositions to cyclone and bag filter efficiency and further on to packing, and of course, final powder characteristics and properties [1,2].

The fluid flows into the rotary wheel nearly at the center, from here the fluid enters narrow channels or bushings. From the entrance into the bushings to the exit of the atomizer the fluid and the air is accelerated towards the exit due to the centrifugal force and is at the same subject to a substantial Coriolis force. In addition, there are internal forces within the fluids e.g., surface tension and viscous forces. The combination of these forces and the complex geometry makes understanding of the fluid flow within a rotary atomizer extremely difficult.

Computational Fluid Dynamics (CFD) as a numerical method for solving the equations of motion gives an opportunity to directly simulate the flow under various operating conditions and various geometries. This will be the focus of this paper and will be thoroughly elaborated in the next section. Previous studies where CFD is used to model various aspects of spray drying includes $[3,4,5,6]$.

The results obtained from this study is then compared to existing in-house atomization experiments where the power usage and droplet size distribution are measured. Furthermore, it should be noted that this study is a continuation of previous studies [7,8,9,10]. Here information about the method used in the experiments, that are referred to in this study, can be found.

\section{Materials and Methods}

Albeit, several different wheels have been modelled, and results will be presented accordingly. We will, in this paper, only present the CFD-model for one geometry. We will, however, present results for other geometries since these have been simulated in a similar way. The differences in geometry for the rotary atomizer wheels presented in this paper consists of different diameters of the rotary wheel, different number of bushings in the rotary wheel, as well as slight deviations in the geometry of the cavity of the wheel. 
One of the wheels modelled consists of eight bushings equally distributed over the circumference of the wheel. Hence, symmetry considerations allow us to model a subdomain of the wheel, i.e., $\theta \in[-\pi / 8: \pi / 8]$ with a bushing at the center of the domain, i.e., $\theta=0$, where $\theta$ represents an angle (rad).

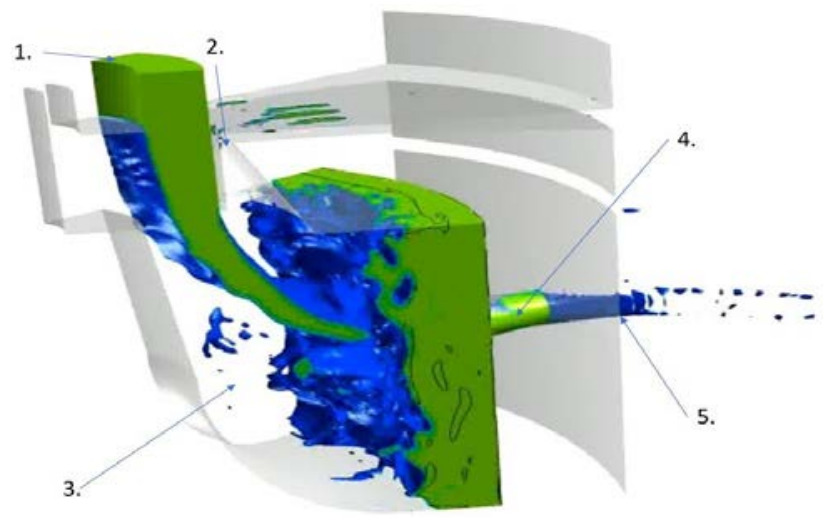

Fig. 1 Illustration of the geometry of the simulated part of a wheel. Indicated by numbers and arrows are 1) inlet, 2) overflow, 3) cavity, 4) bushing 5) exit. Notice, how water enters through the inlet and exits through the overflow and the bushing.

In Fig. 1 the geometry of the modelled domain is shown. The modelled domain consists of the inlet, the wheels cavity of the wheel, the bushing, the 'overflow area' and the outside of the bushing, i.e., the drying chamber.

To give realistic flow distributions to the inlet, the flow in the liquid distributor prior to the inlet has, although not shown in this paper, been modelled for different feed rates. The solution to these prior investigations have been used as boundary conditions for the inlet flow.

Under normal operating conditions the fluid will move from the inlet through the cavity of the wheel, through the bushing and out into the drying chamber. Under abnormal operating conditions, i.e., very high values of the feed rate, part of the fluid will exit the wheel through the overflow. In the small part of the drying chamber included in this model, the breakup of the exiting jet and thin film annulus is modelled. Here the resulting drops are accounted for and the size distribution a size distribution is calculated.

All simulations presented in this study have been performed using ANSYS Fluent with a volume of fluid (VOF) approach.

\subsection{Governing equations}

The governing equations for fluid and the air are the Navier-Stokes equation and continuity equation of fluid and air where subscript $j$ represents one of the fluid phases air (a) or water 
(w) and $\varepsilon$, is the fraction of component j. Note that there are only two phases - hence, $\varepsilon_{a}+$ $\varepsilon_{w}=1$.

$$
\begin{gathered}
\frac{\partial}{\partial t}\left(\varepsilon_{j} \rho_{j}\right)+\nabla \cdot\left(\varepsilon_{j} \rho_{j} \boldsymbol{u}\right)=0 \\
\frac{\partial}{\partial t}\left(\varepsilon_{j} \rho_{j} \boldsymbol{u}\right)+\nabla \cdot\left(\varepsilon_{j} \rho_{j} \boldsymbol{u u}\right)=-\varepsilon_{j} \nabla p+\nabla \cdot\left(\varepsilon_{j} \boldsymbol{\tau}_{j}\right)+\varepsilon_{j} \rho_{j} \boldsymbol{g}+\boldsymbol{f} .
\end{gathered}
$$

Here, $\boldsymbol{u}$, is the velocity field $(\mathrm{m} / \mathrm{s}), \rho$, is the density $\left(\mathrm{kg} / \mathrm{m}^{3}\right), \varepsilon$, is the volume, $p$ is the pressure (Pa) and $\boldsymbol{g}$, is the gravitational acceleration $\left(\mathrm{m} / \mathrm{s}^{2}\right)$ and $\boldsymbol{f}\left(\mathrm{N} / \mathrm{m}^{3}\right)$ represents forces per unit volume, not accounted for by the motion of the fluid, i.e., centrifugal force, surface tension and the Coriolis force. Here, the stress tensor, $\boldsymbol{\tau}_{j}$, is given by

$$
\boldsymbol{\tau}_{j}=\mu_{j}\left(\left[\nabla \boldsymbol{u}+\nabla \boldsymbol{u}^{T}\right]-\frac{2}{3}(\nabla \cdot \boldsymbol{u}) \boldsymbol{I}\right)
$$

where, $\mu$, is the viscosity (Pa·s) and $\boldsymbol{I}$ represents the unit matrix. Note that the water is modelled as incompressible whereas the air is compressible. Turbulence is modelled with a RANS $(\mathrm{k}, \omega)$ model.

\subsection{Boundary conditions}

\subsubsection{Walls}

On the walls we impose a no flux condition in direction of the normal vector and a no-slip condition in tangential direction. I.e., there is no transport of fluid through solid walls and the relative velocity between the fluid and a solid wall is zero.

\subsubsection{Outlet}

At the surface of the domain where the fluid exits into the drying chamber a zero gauge pressure boundary condition is imposed.

\subsubsection{Periodic boundary conditions}

At all boundaries normal in the direction of the rotation has a periodic boundary, i.e., what exits on one side enters on the other. Hence,

$$
\alpha\left(r,-\frac{\theta}{8}, z\right)=\alpha\left(r, \frac{\theta}{8}, z\right) .
$$

Here $\alpha$ represents conserved properties, i.e., velocity field, $\boldsymbol{u}$, the pressure, $p$, the volume of fluid fractions, $\varepsilon_{f}$, as well as the turbulence parameters $k$ and $\omega$. 


\subsubsection{Inlet}

As mentioned the velocity field at the inlet for different volumetric inlet fluxes, $\dot{V}\left(\mathrm{~m}^{3} / \mathrm{s}\right)$ and different geometries, $\Omega$, has been determined in a previous study. The velocity field, $v(\mathrm{~m} / \mathrm{s})$ at the inlet for different feed flows are used as a boundary condition at the inlet. Hence,

\subsection{Mesh}

$$
\boldsymbol{u}_{i}=\boldsymbol{v}_{i}(\dot{V}, \Omega)
$$

The mesh used is a swept mesh with the special ability of adapting size according to the gradient of volume of fluid, i.e., the mesh has small elements in the interface between fluid and air. This adaptive mesh ensures that the resolution of the air-water interface improved without compromising the simulation time. An illustration of the mesh and the size adaption is shown in Fig. 2.

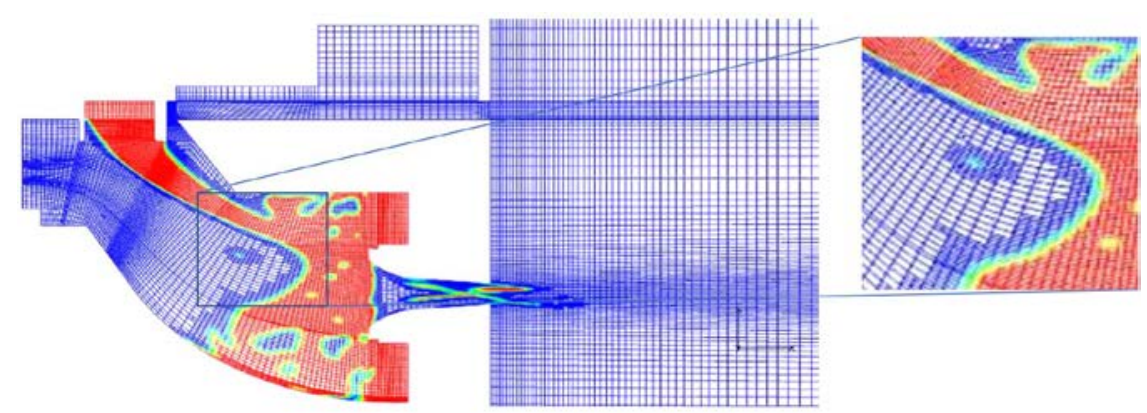

Fig. 2 Illustration of the mesh as well as how the mesh is refined depending on the VOF-gradient. The red part of the figure represents water and the blue is air.

\subsection{Drop size calculations}

To estimate the drop size in the simulations, a virtual surface is included at a given distance from the bushing exit. The volume of fluid droplets that passes through this virtual surface is then directly related to the volume of a sphere and the radius is calculated accordingly. The observation of droplets passing through the virtual surface is collected for enough time to give a reasonable size distribution.

\section{Results and discussions}

As mentioned in the introduction the results from the CFD-simulations are compared to experimental results. Firstly, we shall relate the findings from the simulations to experimental power consumptions. Secondly, we shall relate the droplet size distribution from the simulations to experimental results.

\subsection{Power consumption and wheel capacity}

The equation governing the power consumption is given by,

$$
P=\dot{m}_{b}\left(\omega R_{b}\right)^{2}=\left(\dot{m}_{f}-\dot{m}_{o}\right)\left(\omega R_{b}\right)^{2} .
$$


Here, $P$ is the power $(\mathrm{W}), \dot{m}_{b}$, is the liquid mass-flux $(\mathrm{kg} / \mathrm{s})$ through the bushing, $\omega$ is the angular velocity (rad/s), $R_{b}(\mathrm{~m})$ is the radius at the bushing exit, $\dot{m}_{f}$ is the mass-flux of feed (kg/s) and, $\dot{m}_{o}$ is the mass-flux of liquid through the overflow $(\mathrm{kg} / \mathrm{s})$. Hence, a linear dependency of power versus inlet flux is expected if no fluid passes through the overflow, i.e., $\dot{m}_{o}=0$. Furthermore, a larger power consumption is needed for larger angular velocities of the rotary atomizer. Trivially, the linear dependence between power and feed flow ceases to exist when fluid passes through the overflow - which will happen at a sufficiently high inlet-fluxes.

It is, however, not trivial that simulations from CFD and experiments performed on the wheels simulated are able to produce overflow for the same values of the influx.

The power distribution is a function of inlet flux, both experimental and from simulations, are presented in Fig. 3 for three different angular velocities. As seen there is a good agreement between experiment and simulations. For small values of the inlet flux there is a linear tendency between power consumption and inlet flux. Increasing the inlet flux even further causes a higher fraction of the fluid to pass through the 'overflow' inducing a larger deviation from the straight line.

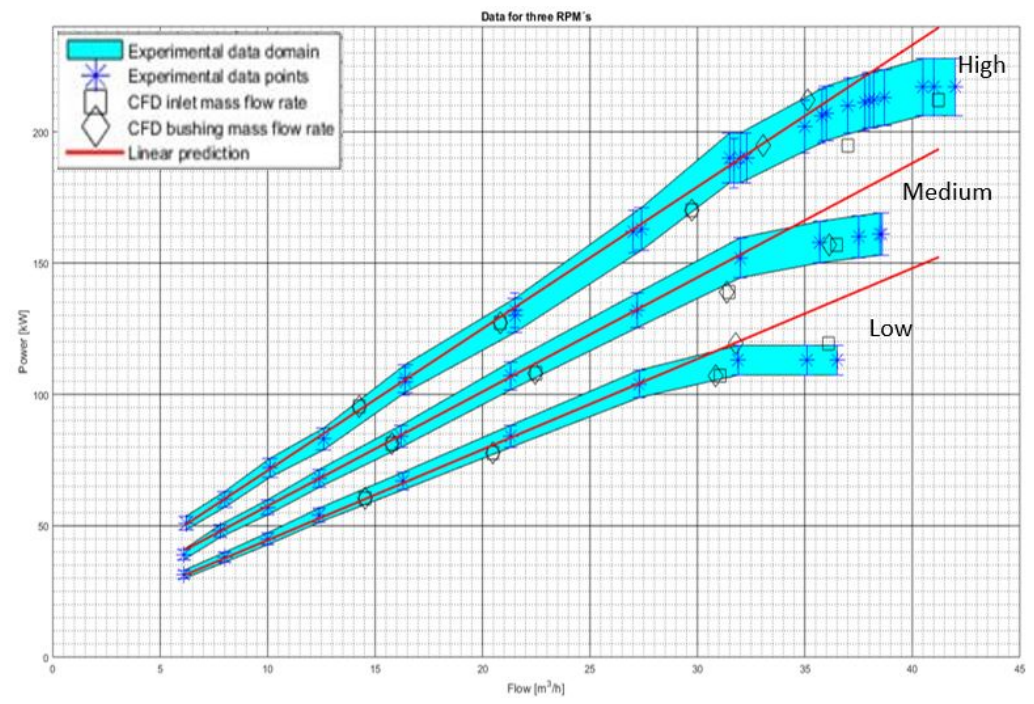

Fig. 3 Power consumption as a function of feed-rate for CFD simulations and experimental data for three different RPM - increasing from bottom to top.

It can also be seen from Fig. 3 that the critical inlet flux is increasing for increasing angular velocity of the wheel. This is expected due to the larger acceleration of the fluid in radial direction due to an increase in centrifugal force. As seen from the figure there is an excellent agreement between the CFD simulations and experiments made on the wheel simulated at 
corresponding angular velocities. In figure Fig. 1 it is illustrated how fluid passes through the overflow during simulations.

\subsection{Prediction of atomization and droplet size distribution}

Experimental results of the drop-size distribution as well as measured drop-size distribution from the simulations are shown in Fig. 4. As seen there is a good qualitative agreement between the two. The droplet-size depends among other things on the exiting jet from the bushing. The exiting jet and breakup from a simulation and experiment are shown in Fig. 5.

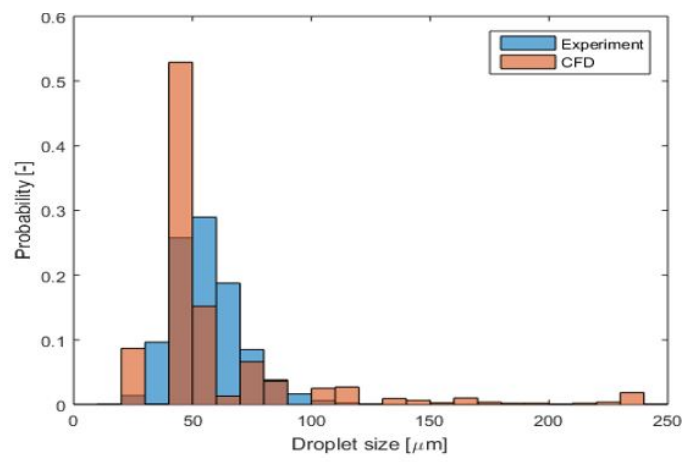

Fig. 4 Droplet size distribution from CFD simulations and experimental results measured at 110 mm from the bushing exit at an angular velocity of 29000 RPM.

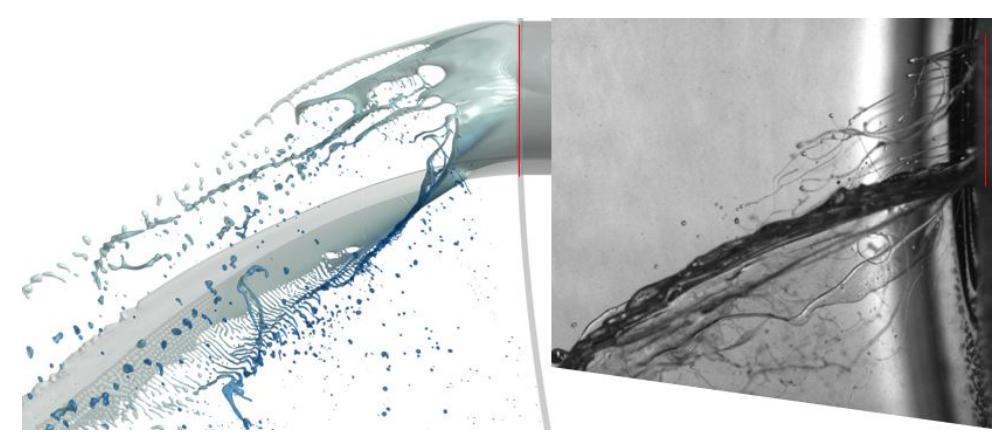

Fig. 5 Snapshot from the CFD-simulations (left) and experiment (right) of the breakup of the thin film and exiting jet into droplets.

The ability to reproduce experimental results serves as a validation of the model. This validation is a first step for future research, where extraction of valuable information, e.g., total wall shear stress, mixing, rivulet height, film thickness for different fluids and different operating conditions. This in return allows for topology optimization, power consumption and essentially optimized conditions for drying and end-product.

The inclusion of CFD in our production line allow us to design new geometries for rotary wheels, and operate them at optimal feed rates and angular velocities in terms of power 
consumption. Furthermore, the ability to estimate the droplet size distribution for any given fluid makes it possible to give optimal conditions for the final product. Hence, enables us to produce tailor made solutions for a given product and increasing value for industry.

\section{Conclusions}

The CFD model presented in this study gives valuable insight in the fluid flow and interaction between fluid and air in a rotary atomizer. The model can reproduce experimental results for power consumption and droplet size-distribution.

\section{References}

[1] Lefebvre, A.H. Atomization and Sprays; Combustion: An International Series. Hemisphere Publishing Corporation, 1989.

[2] Masters, K. Spray Drying Handbook; Longman Scientific \& Technical, Essex, England, 1991.

[3] Ullum, T.; Sloth, J.; Brask, A. Predicting Spray Dryer Deposits by CFD and an Empirical Drying Model. Drying Technology 2010, 28(5), 723-729.

[4] Huang, L.X.; Kumar, K.; Mujumdar, A.S. A comparative study of a spray dryer with rotary disc atomizer and pressure nozzle using computational fluid dynamic simulations. Chemical Engineering and Processing: Process Intensification 2006, 45(6), 461-470.

[5] Woo, M.W.; Daud, W.R.W.; Mujumdar, A.S.; Wu, Z.; Talib, M.Z.M.; Tasirin, S.M. CFD Evaluation of Droplet Drying Models in a Spray Dryer Fitted with a Rotary Atomizer. Drying Technology 2008, 26(10), 1180-1198.

[6] Gianfrancesco, A.; Turchiuli, C.; Flick, D.; Dumoulin, E. CFD Modeling and Simulation of Maltodextrin Solutions Spray Drying Control Stickiness. Food and Bioprocess Technology 2010, 3(6), 946-955.

[7] Kuhnhenn, K.; Oberthür, A.; Roisman, I.V.; Joensen, T.V.; Tropea, C. Droplet Size Determination on a Rotary Atomizer Wheel with Time-Shift Technique in Comparison to Analytical Theory. ILASS - Europe 2016, 27'th Annual Conference on Liquid Atomization and Spray Systems, 4-7 September 2016, Brighton, UK.

[8] Kuhnhenn, K.; Luh, M.; Joensen, T.V.; Roisman, I.V.; Tropea, C. Experimental Characterization of Spray generated by a Rotary Atomizer Wheel. ILASS - Europe 2017, 28'th Annual Conference on Liquid Atomization and Spray Systems, 6-8 September 2017, Valencia, Spain.

[9] Kuhnhenn, K.; Roisman, I.V.; Tropea, C.; Joensen, T.V. Study of the internal flow in a rotary atomizer and its influence on the resulting spray. 9th World Conference on Experimental Heat Transfer, Fluid Mechanics and Thermodynamics, 12-15 June 2017, Iguazu Falls Brazil.

[10] Kuhnhenn, K.; Joensen, T.V.; Reck, M.; Roisman, I.V.; Tropea, C. Study of the internal flow in a rotary atomizer and its influence on the properties of the resulting spray. International Journal of Multiphase Flow 2018, 100, 30-40. 